\title{
A METHODOLOGY FOR FACTOR SCREENING FOR MULTIPLE RESPONSE COMPUTER SIMULATION MODELS
}

\author{
Lori S. Cook \\ Department of Industrial Engineering Technology \\ Southern College of Technology \\ Marietta, Georgia 30060, U.S.A
}

\begin{abstract}
The majority of the literature dealing with simulation output analysis has focused on single response models with a limited number of input variables. However, most real world simulation applications involve multiple performance measures and numerous input variables. The purpose of this session is to outline a methodology for factor screening in the presence of multiple simulation responses. The intent is to provide general guidelines to reducing the original set of $n$ input factors to a subset of $k$ factors which exert the most significant impact on a set of $m$ performance measures.
\end{abstract}

\section{INTRODUCTION}

Simulation is defined as the development of a mathematical/logical model of a system, and the experimental manipulation of that model on a digital computer. In simplified form, a simulation model can be thought of as a "black box" into which a set of $n$ controllable factors are combined to generate a set of $m$ response values. The response values correspond to system performance measures which are to be evaluated. Typically, only a subset of the input factors are truly important. The difficulty lies in the determination of which input factors are important. The analyst does not want to expend unnecessary simulation runs analyzing factors which are unimportant.

Due to the complexity of systems modeled, the analyst is usually interested in the effect of the input changes on multiple performance measures. Techniques are available to screen input factors for single response simulation models. However, techniques are currently not available to address factor screening in the presence of multiple output responses. The mul- tiple-response nature of a simulation model adds a new dimension of complexity when attempting to screen input factors. When screening, an input factor may be important for one response measure, but may not exhibit importance for another response measure. The analyst must determine if the factor should be screened or retained.

\section{REVIEW OF FACTOR SCREENING FOR SIMULATION ANALYSIS}

Factor screening is the process of determining the subset of factors in a simulation model which exert the greatest impact on the set of response variables. Mauro and Smith (1984) stated the following goals of factor screening: (1) to classify as important as many of the truly important factors as possible, (2) to avoid declaring unimportant factors as important, and (3) to accomplish these objectives using the smallest number of simulation runs possible. The experimental design strategy selected for factor screening will depend on the level of saturation of the design.

\section{FACTOR SCREENING METHODOLOGY}

After the selection of the appropriate experimental design, the following methodology is used to assess the model in the presence of multiple output responses.

1. Perform simulation trials at each of the specified design points in the experimental design.

2. Construct a data set of simulation results for the responses $\mathrm{y}_{\mathrm{j}}, \mathrm{j}=1, \ldots, \mathrm{m}$.

3. Develop multiple regression equations for each of the system responses using the coded experimental design matrix as values for the input factors $x_{i}, i=$ $1, \ldots, n$. 
4. Check the F-value and Prob $>F$ of each multiple regression equation to ensure that the model explains a significant portion of the variation in the data.

5. Perform t-tests for each regression parameter estimate $b_{i, j}, i=1, \ldots, n$ and $j=1, \ldots, m$. Determine if the individual parameter estimates are significantly different from zero.

6. Develop a significance matrix to assess the frequency $f_{i j}$ with which an input factor is significant over all response measures $y_{j}, j=1, \ldots, m$.

7. Construct a matrix of the regression parameter estimates $b_{i, j}$ for each system response $y_{j}$.

8. Construct a matrix showing the percent contribution of each parameter to the summation of the parameters for each output response.

9. Develop a cellular group matrix showing percent contribution measures of the input factors and responses.

10. Develop a weighted composite regression function using different weighting schemes.

11. Develop a ranking of input parameters to assess potential candidates for screening based on t-test, weighted regression composite function and weighted group regression composite function.

\section{CONCLUSIONS AND RECOMMENDATIONS}

Factor screening should only be used in certain simulation modeling situations. The appropriate use of factor screening is a function of the number of factors involved, the number of simulation runs required, the number of simulation runs available for use and the available time for analysis.

The factor screening methodology discussed should only be applied to determine the subset of important factors within the simulation model. It is not intended for predicting the actual values of the performance measures. Therefore, the simulationist should use the minimum number of runs necessary for screening to achieve the desired accuracy he or she is willing to accept.

The number of input factors dictates if there is a need for factor screening analysis. If the number of input factors is relatively small, factor screening is not usually necessary. If factor screening is employed for small $n$, it would be more appropriate to use a full factorial or $2^{k-p}$ fractional factorial design. However, if there are a large number of input factors, it would be more appropriate to use a screening methodology to determine the subset of factors which are most significant. By screening the input parameters, one can also determine the level of accuracy required for the significant input parameters. Hence, for a significant input factor parameter the data estimates should have a higher degree of accuracy for the next phase of the simulation model.

The methodology proposed is only recommended for the initial factor screening of input factors. It is not intended to be used for predictive purposes. After the screening experiments, the next stage of the simulation analysis would include the optimization of the model. Potentially in this stage, the previous simulation runs could be augmented. In addition, it is recommended that in subsequent experimentation those factors which were deemed insignificant should be retained in the model and set at their respective midpoint levels. The methodology developed is viewed as an initial starting point in analyzing factor screening designs for multiple response simulation models.

\section{REFERENCES}

Cook, L. S. 1992. Factor Screening Designs For Multiple Response Computer Simulations. Unpublished Dissertation, Department of Industrial Engineering, University of Louisville.

Mauro, C.A. and Smith D.E. 1984. Factor Screening in Simulation: Evaluation of Two Strategies Based on Random Balance Sampling. Management Science, 30(2):209-221.

\section{AUTHOR BIOGRAPHY}

LORI S. COOK is an Assistant Professor in the Department of Industrial Engineering Technology at Southern College of Technology. She received her B.S., M.Eng. and Ph.D. in Industrial Engineering from the University of Louisville. She has worked for Wright Patterson Air Force Base, Armco Eastern Steel Division and Kentucky Fried Chicken Corporation where she performed simulation analysis of complex systems. Her research interests include the application of optimization and simulation modeling to complex systems. She is a member of Alpha Pi Mu, Tau Beta Pi, IIE TIMS, and ORSA. 